\title{
Suchtrisiko: Biologie und Umwelt
}

\author{
An der Entwicklung einer Alkoholabhängigkeit sind \\ Umweltfaktoren etwa gleichrangig beteiligt wie die \\ Biologie. Das bestätigen aktuelle Daten einer großen \\ schwedischen Adoptionsstudie.
}

Dass das Risiko, alkoholabhängig zu werden, wie bei anderen Drogen etwa zur Hälfte genetisch bedingt ist, belegen bereits Ende des letzten Jahrhunderts durchgeführte Zwillingsstudien. Sie hatten genetisch identische eineiige Zwillinge mit zweieiigen, also zu $50 \%$ genetisch identischen Zwillingen verglichen, die in derselben familiären Umwelt aufgewachsen waren [z. B. Prescott CA et al. Am J Psychiatry 1999; 156: 34 -40], erklärte Professor Andreas Heinz, Direktor der Klinik für Psychiatrie und Psychotherapie an der Charité, Berlin. Als eine der Risikofaktoren nannte Heinz eine hohe „Trinkfestigkeit“ aufgrund eines fehlenden Warnsignals bei akutem Alkoholmissbrauch. Diese ist neuesten Erkenntnissen nach an ein Gen (ALDH1A2) gebunden, das die Metabolisierung des Alkohols beeinflusst. Wie Heinz erläuterte, war bei Menschen mit riskantem Alko- holkonsum eine verminderte Kontrolle des Alkoholkonsums mit der Methylierung an einer anderen Stelle desselben Gens korreliert [Harlaar N et al. ACER 2014; 38: 713-21]. Für den Grad der Methylierung und damit die Ablesbarkeit der Gene sind disponierende Umweltfaktoren mit verantwortlich, darunter frühe Stresserfahrungen, die letztlich die Kontrolle über den Alkoholkonsum herabsetzen, erklärte Heinz. Die Ergebnisse einer 2015 veröffentlichten Adoptionsstudie mit über 18.000 Personen bestätigen nun eindrucksvoll, dass Alkoholabhängigkeit, Drogenkonsum und weitere soziale Probleme der biologischen (1,46-fach) und Adoptiveltern (1,40-fach) bei den Nachkommen das Risiko für Alkoholabhängigkeit oder schädlichen Alkoholkonsum erhöhen [Kendler KS et al. JAMA Psychiatry 2015; 72: $211-8$ ] - das bedeutet, genetische und Umweltfaktoren inklusive der Erziehung und des familiären Umfelds sind fast gleichrangig an der Entwicklung eines klinisch relevanten Alkoholproblems beteiligt. Ute Ayazpoor, freie Medizinjournalistin

Abhängigkeit und Sucht (Andreas Heinz, Berlin).

Psychiatrie Update, 11. - 12.3.2016, Wiesbaden

\section{Rückfallprophylaxe bei Alkoholabhängigkeit}

\author{
Zum Standard bei der Behandlung der Alkoholab- \\ hängigkeit gehört neben dem qualifizierten Entzug \\ auch die Rückfallprophylaxe. Hier bietet sich eine \\ Verhaltenstherapie - eventuell mit medikamentöser \\ Unterstützung - an.
}

Aktuell sind in Deutschland Acamprosat und Naltrexen für die Rückfallprophylaxe bei Alkoholabhängigkeit zugelassen, berichtete Professor Andreas Heinz, Berlin. Beide Medikamente werden im Zusammenhang mit psychosozialen Therapieprogrammen eingesetzt und nutzen unterschiedliche Strategien: Acamprosat verlängert die Abstinenzzeiten und Naltrexon reduziert die Trinkmenge. Beide helfen laut einer Erhebung einem von zehn behandelten Patienten, abstinent zu bleiben [Müller CA et al. Eur Neuropsychopharmacol 2015; 8: 1167 - 77] - es besteht aber weiterhin Bedarf an wirksamen Behandlungsoptionen, so Heinz.

\section{Gaba-B-Agonist reduziert Rückfallrisiko}

Eine wirksame Option könnte etwa das in dieser Indikation nicht zugelassene Baclofen sein. Der Gaba-B-Agonist wurde in einer Studie in der Dosierung zwischen $30 \mathrm{mg}$ und $270 \mathrm{mg}$ nach individuellem Bedarf über einen Zeitraum von drei Monaten eingesetzt und reduzierte das Rückfallrisiko gegenüber Placebo signifikant. Wie Heinz berichtete, wurden 15 von 22 Alkoholkranken unter Placebo rückfällig, aber nur fünf von 21 unter variabel dosiertem Baclofen. Die kumulative Dauer der Abstinenz lag unter Baclofen bei knapp 68 Tagen gegenüber knapp 52 Tagen unter Placebo ( $\triangleright$ Abb. 1). Heinz: „Leider kann das Me-

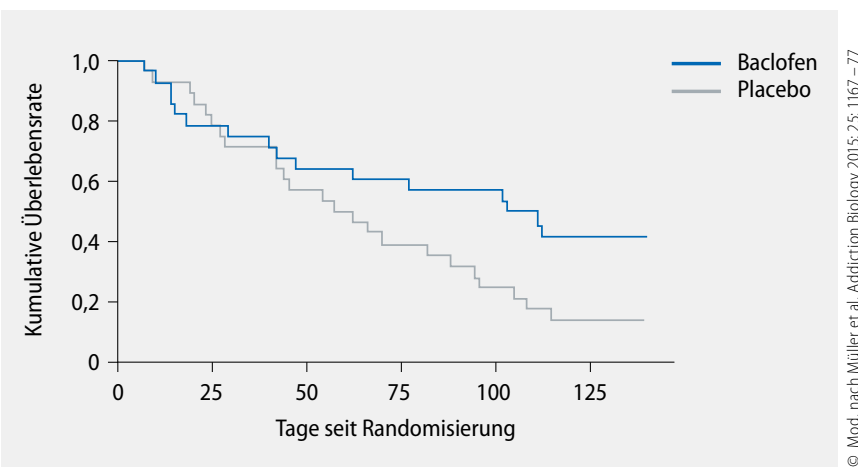

1 Anteil der Patienten („cumulative Survival“, die im Beobachtungszeitraum (Tage seit Randomisierung) abstinent blieben $($ Baclofen $=40 \%)$, Placebo $=20 \%)$

dikament nicht verordnet werden. Noch fehlen zudem weitere Studien für eine Zulassung."

\section{Verhaltenstraining wirkt längerfristig}

Längerfristige Abstinenz ist gegebenenfalls durch ein Verhaltenstraining gegen gewohnheitsmäßigen Alkoholkonsum zu erreichen, erklärte Heinz. Hochinteressant sei ein Verfahren, mit dem die gewohnheitsmäßige Zurückweisung von Alkohol antrainiert werde, um automatisierte Tendenzen, Alkohol zu konsumieren, abzubauen. Dabei werden Menschen neutrale Bilder und Alkoholbilder im Hoch- und Querformat angeboten und sie werden aufgefordert, Bilder im Querformat von sich wegzu- 